\title{
Synchronous renal cell carcinoma metastasis to the contralateral adrenal gland and pancreas: A case report with 7-year follow-up subsequent to surgical therapy
}

\author{
CUNZAO WU ${ }^{1}$, ZHENXU ZHOU ${ }^{1},{\text { XUETING } \text { YE }^{1} \text { and WEILIE HU }}^{2}$ \\ ${ }^{1}$ Transplantation Center, The First Affiliated Hospital of Wenzhou Medical University, Wenzhou, Zhejiang 325003; \\ ${ }^{2}$ Department of Urology, Guangzhou General Hospital of Guangzhou Military Command, \\ Guangzhou, Guangdong 510010, P.R. China
}

Received March 4, 2015; Accepted March 8, 2016

DOI: $10.3892 / \mathrm{ol} .2016 .4510$

\begin{abstract}
Metastatic renal cell carcinoma (RCC) disseminates to a number of organ sites and few patients demonstrate long-term survival following surgery. However, synchronous metastasis of RCC to the contralateral adrenal gland and pancreas is rare. In the present report, a case of synchronous RCC metastasis to the contralateral adrenal gland and pancreas in a 55-year-old patient, with an 116x92x61 mm right renal

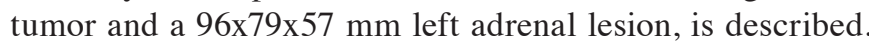
In April 2007, right nephrectomy was performed to treat the RCC, left adrenalectomy was performed to treat the adrenal tumor and the pancreatic metastases were resected. The patient remained alive at the 7-year follow-up appointment.
\end{abstract}

\section{Introduction}

Numerous patients with RCC remain asymptomatic until the late stages of the disease. The prevalence of the classic triad of flank pain, gross haematuria and a palpable abdominal mass is rare, while paraneoplastic syndromes occur in $~ 30 \%$ of patients with symptomatic RCCs. A small number of patients present with symptoms caused by metastatic RCC (1). Metastatic disease at diagnosis is observed in $\sim 25-30 \%$ of patients with renal cell carcinoma (RCC), and $<5 \%$ of patients exhibit solitary metastasis. The most common distant RCC metastasis sites are the lungs (50-60\%), bone (30-40\%), liver (30-40\%) and brain (5\%); however, metastasis may involve virtually any organ site (2). While adrenal metastasis from RCC to the ipsilateral adrenal gland is common, synchronous metastasis of $\mathrm{RCC}$ to the contralateral adrenal gland is rare, and affects only

Correspondence to: Professor Weilie Hu, Department of Urology, Guangzhou General Hospital of Guangzhou Military Command, 111 Liuhua Road, Guangzhou, Guangdong 510010, P.R. China E-mail: weiliehu0515@163.com

Key words: metastatic renal cell carcinoma, contralateral adrenal gland, pancreas
$0.51 \%$ of patients $(6 / 1,179)(3)$. Pancreatic metastases from RCC are additionally rare (accounting for $1.5-3 \%$ of all metastatic RCC cases), and these cases typically present a number of years subsequent to the primary RCC diagnosis $(4,5)$.

For localised RCC, surgery is the only curative treatment with high-quality evidence and for metastatic RCC surgery is curative only if the total tumor burden can be removed (1). As prognosis is directly associated with the stage or degree of tumor dissemination, metastatic RCC generally equates to a poor prognosis for patients; indeed, response rates for treated patients remain low, at $\sim 15-25 \%$, with 5-year survival rates of $5-10 \%$ and overall median survival of $<1$ year (2). However, in certain cases, radical resection of metastases may prolong survival. In the present paper, a case of synchronous RCC metastasis to the contralateral adrenal gland and pancreas is reported. The patient remained alive at the final follow-up appointment, a total of 7 years subsequent to undergoing simultaneous right nephrectomy, left adrenalectomy and segmental pancreatectomy.

\section{Case report}

A 55-year-old man with no relevant medical history visited the Transplantation Center of The First Affiliated Hospital at Wenzhou Medical University (Wenzhou, China) to undergo a routine physical examination in March 2007. Abdominal magnetic resonance imaging (MRI; 1.5T; GE Healthcare, Little Chalfont, UK) revealed a 99x81 $\mathrm{mm}$ right renal tumor and a 94x72 mm left adrenal lesion (Fig. 1). Thoracic computed tomography (CT; LightSpeed Pro 16; GE Healthcare) and bone scans were negative. The patient's full blood count, electrolytes, and liver and renal function were within normal ranges. Metabolic evaluation included 24-h urine collection for assessment of 17-ketosteroids, 17-hydrocorticoids, metanephrines, cortisol and vanillylmandelic acid, the levels of which were normal.

During surgery (April 2007), a bilateral subcostal incision was made. The right renal tumor measured 116x92x61 mm, and the left adrenal lesion measured $96 \times 79 \times 57 \mathrm{~mm}$. A $10 \times 10 \mathrm{~mm}$ nodule on the lower edge of the body of the pancreas was observed. The nodule exhibited a good plane for dissection, which permitted complete excision by segmental 


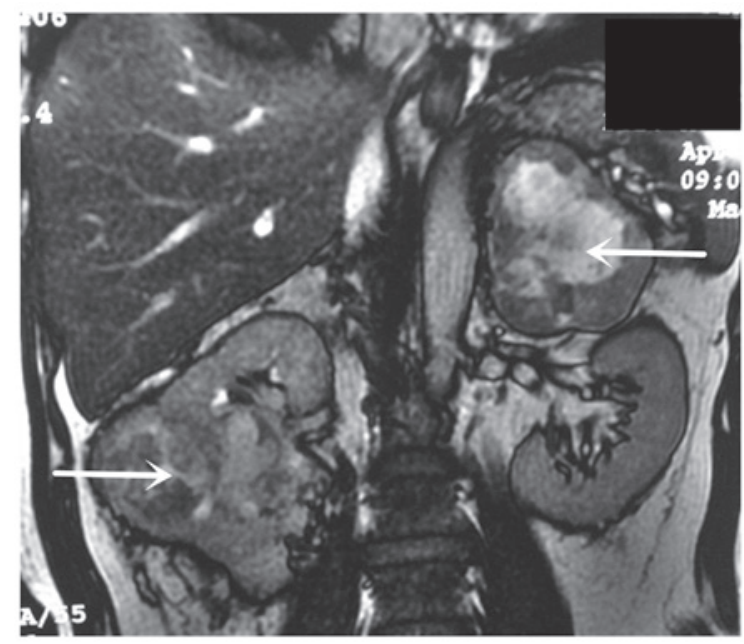

Figure 1. Magnetic resonance imaging reveals a right renal tumor (left arrow) and a left adrenal lesion (right arrow).

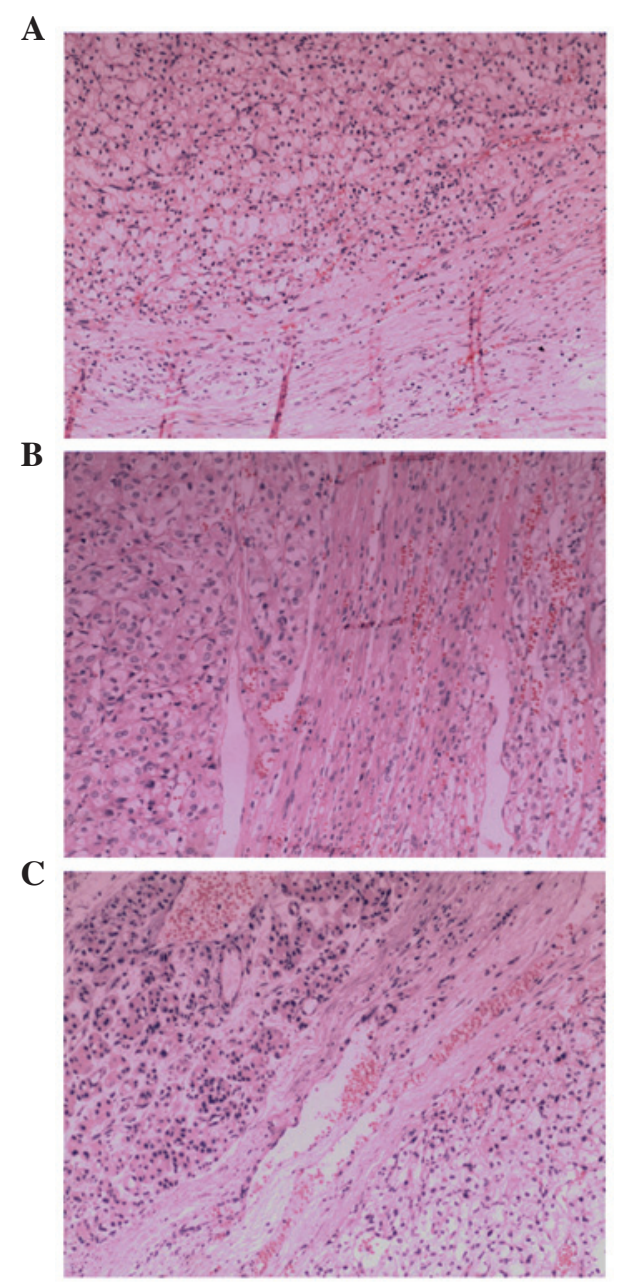

Figure 2. Histopathological examination of tissue samples. (A) Clear cell RCC was observed in the right renal specimen. (B) Metastatic clear cell RCC was observed in the adrenal specimen. (C) Metastatic clear cell RCC was observed in the pancreatic specimen. Hematoxylin and eosin staining; magnification, x100. RCC, renal cell carcinoma.

pancreatectomy. The nodule was fixed in $10 \%$ fixed in formalin, dehydrated in graded alcohol, embedded in paraffin, sliced to a thickness of $5 \mu \mathrm{m}$ and deparaffinized in xylene.
Histopathological examination (hematoxylin and eosin staining) revealed that the contralateral adrenal tumor and pancreatic nodule were pathologically identical to the renal tumor. The histopathological specimens were also used to determine a diagnosis of clear cell RCC (Fig. 2). All surgical margins were tumor-free. It was determined that the clinicopathological stage was pT2bN0M1 (6).

The patient recovered 12 days subsequent to surgery and was discharged from hospital. He was administered interferon immunotherapy (intrahepatic injection with $6 \mathrm{MIU}$ interferon alfa-2b iinjection 3 times per week) for 6 months. CT at 24 months post-surgery identified no evidence of recurrence or new metastatic lesions. However, abdominal CT 51 months subsequent to surgery revealed 3 lesions in the bilateral adrenal area. A total of 66 months later, the patient exhibited 7 metastatic nodules in the bilateral lungs, including 4 in the left lung and 3 in the right. The patient refused additional treatment and remained alive with these metastatic lesions during the annual follow-up appointments for 7 years. The patient succumbed on November 30, 2015.

Written informed consent was obtained from the patient for the publication of the present case report and accompanying images, and ethical approval was obtained from the ethics committee of the First Affiliated Hospital of Wenzhou Medical University.

\section{Discussion}

To the best of our knowledge, the present study describes the first reported case of synchronous metastasis to the contralateral adrenal gland and pancreas from RCC. The fact that this patient remained alive at the final follow-up appointment, a total of 7 years subsequent to the surgical resection of the primary tumor and metastases, is encouraging for treatment of similar cases in the future.

$\mathrm{RCC}$ is known for its multiple modes of presentation and its propensity to metastasize to almost every organ (2). Metastasis of RCC to the pancreas may occur via the hematogenous route (involving the draining collateral veins from the primary RCC lesion) or via the lymphatic route (involving the retrograde lymph flow through the retroperitoneal nodes), and direct spread to the pancreas is rare (7). The pathway through which metastasis to the contralateral adrenal gland occurs remains to be elucidated, although the antegrade hematogenous route may be involved (8). Notably, previous studies have demonstrated that contralateral adrenal metastases are able to grow to a considerable size, even when originating from only a small number of metastatic RCC cells, and without metastasizing to other organs; therefore, the adrenal gland may have an increased affinity for RCC spread compared with other organs $(9,10)$.

Diagnosis of adrenal metastases from RCC typically relies on findings from CT and ultrasonography examinations (11). Even with these advanced imaging technologies, it can be difficult to differentiate adrenal metastases from primary tumors of the adrenal gland, for example, adrenal cortical adenoma (12). It is generally considered that radiological findings of a solitary adrenal mass in the absence of elevated serum adrenocortical hormones is highly suggestive of an adrenal metastatic lesion, and the present case confirmed this (13). As conservative treatments, including chemotherapy, hormone 
therapy and radiotherapy, have not proven effective in the treatment of metastatic RCC, surgical removal of solitary metastatic lesions remains the only treatment option for such patients (14). Surgical resection of adrenal metastases is safe, and additionally promoted by the development of a laparoscopic technique, using either a retroperitoneal or a transperitoneal approach (15). In the treatment of the current case, the ipsilateral adrenal gland was spared during radical nephrectomy in order to avoid causing postoperative adrenal insufficiency; this strategy was designed in accordance with previous findings that have suggested that a routine ipsilateral adrenalectomy is not recommended when there are no suspicions of tumor spread (16).

If the pancreatic metastases from RCC are small and isolated, patients may be asymptomatic (17). However, a larger pancreatic tumor may cause abdominal pain, jaundice and weight loss (18). In the present case, the pancreatic metastatic nodule was small and not detected during preoperative examination. As pancreatic metastases from RCC are typically isolated lesions, they are more amenable to surgical treatment (19). The type of pancreatic metastasectomy selected for treatment, including pancreaticoduodenectomy middle-segment or distal pancreatectomy, depends upon the location of the tumor within the pancreas, and the decision should be made with the goal of achieving clear margins of resection (12). Current pancreatic resections have low rates of mortality and morbidity, particularly when performed at large healthcare facilities (20).

In conclusion, metastatic RCC is generally associated with a poor prognosis (2); few patients experience long-term survival following surgery, and the clinical application of targeted drugs, including interferon immunotherapy, may improve patient survival (21). In the current case, the patient underwent simultaneous right nephrectomy, left adrenalectomy and segmental pancreatectomy, followed by receipt of interferon immunotherapy for 6 months. The patient survived for $>7$ years after undergoing treatment. Thus, complete surgical excision of the primary tumor and metastases may be the best available treatment for certain patients.

\section{Acknowledgements}

The present study was funded by the Zhejiang Provincial Natural Science Foundation of China (grant no. LY12H10004).

\section{References}

1. Ljungberg B, Bensalah K, Canfield S, Dabestani S, Hofmann F, Hora M, Kuczyk MA, Lam T, Marconi L, Merseburger AS, et al: EAU guidelines on renal cell carcinoma: 2014 update. Eur Urol 67: 913-924, 2015.

2. Gupta K, Miller JD, Li JZ, Russell MW and Charbonneau C: Epidemiologic and socioeconomic burden of metastatic renal cell carcinoma (mRCC): A literature review. Cancer Treat Rev 34: 193-205, 2008.
3. Antonelli A, Cozzoli A, Simeone C, Zani D, Zanotelli T, Portesi E and Cosciani Cunico S: Surgical treatment of adrenal metastasis from renal cell carcinoma: A single-centre experience of 45 patients. BJU Int 97: 505-508, 2006.

4. Sellner F, Tykalsky N, De Santis M, Pont J and Klimpfinger M: Solitary and multiple isolated metastases of clear cell renal carcinoma to the pancreas: An indication for pancreatic surgery. Ann Surg Oncol 13: 75-85, 2006.

5. Zacharoulis D, Asopa V, Karvounis E and Williamson RC: Resection of renal metastases to the pancreas: A surgical challenge. HPB (Oxford) 5: 137-141, 2003.

6. Kim SP, Alt AL, Weight CJ, Costello BA, Cheville JC, Lohse C, Allmer C and Leibovich BC: Independent validation of the 2010 American Joint Committee on Cancer TNM classification for renal cell carcinoma: Results from a large, single institution cohort. J Urol 185: 2035-2039, 2011.

7. Sotiropoulos GC, Lang H, Liu C, Brokalaki EI, Molmenti E and Broelsch CE: Surgical treatment of pancreatic metastases of renal cell carcinoma. JOP 6: 339-343, 2005.

8. Moslemi MK, Saghafi H and Firoozabadi MH: Renal cell carcinoma with simultaneous bilateral adrenal metastasis: Ipsilateral radical nephrectomy with contralateral adrenal preservation. Case Rep Oncol 3: 372-379, 2010.

9. Sagalowsky AI, Kadesky KT, Ewalt DM and Kennedy TJ: Factors influencing adrenal metastasis in renal cell carcinoma. J Urol 151: 1181-1184, 1994.

10. Dieckmann KP, Wullbrand A and Krolzig G: Contralateral adrenal metastasis in renal cell cancer. Scand J Urol Nephrol 30: 139-143, 1996.

11. Israel GM and Bosniak MA: How I do it: Evaluating renal masses. Radiology 236: 441-450, 2005.

12. Lau WK, Zincke H, Lohse CM, Cheville JC, Weaver AL and Blute ML: Contralateral adrenal metastasis of renal cell carcinoma: Treatment, outcome and a review. BJU Int 91: 775-779, 2003.

13. Stein A, Mecz Y, Sova Y, Lurie M and Lurie A: Synchronous and metachronous contralateral adrenal metastases from primary renal carcinoma. Urol Int 58: 58-60, 1997.

14. Sakorafas GH, Milingos D, Revelos K, Siafakas J, Kontopoulos P and Peros G: Isolated metachronous contralateral adrenal metastasis from renal cell carcinoma. Mt Sinai J Med 73: 822-824, 2006.

15. Shonkwiler RJ and Lee JA: Laparoscopic retroperitoneal adrenalectomy. Surg Laparosc Endosc Percutan Tech 21: 243-247, 2011.

16. Peters I, Hora M, Herrmann TR, von Klot C, Wegener G, Stransky P, Hes O, Kuczyk MA and Merseburger AS: Incidence of synchronous and metachronous adrenal metastases following tumor nephrectomy in renal cell cancer patients: A retrospective bi-center analysis. Springerplus 2: 293, 2013.

17. Ballarin R, Spaggiari M, Cautero N, De Ruvo N, Montalti R, Longo C, Pecchi A, Giacobazzi P, De Marco G, D'Amico G, et al: Pancreatic metastases from renal cell carcinoma: The state of the art. World J Gastroenterol 17: 4747-4756, 2011.

18. Gilani SM, Tashjian R, Danforth R and Fathallah L: Metastatic renal cell carcinoma to the pancreas: diagnostic significance of fine-needle aspiration cytology. Acta Cytol 57: 418-422, 2013.

19. Lavu H and Yeo CJ: Metastatic renal cell carcinoma to the pancreas. Gastroenterol Hepatol (NY) 7: 699-700, 2011.

20. Wente MN, Kleeff J, Esposito I, Hartel M, Müller MW, Fröhlich BE, Büchler MW and Friess H: Renal cancer cell metastasis into the pancreas: A single-center experience and overview of the literature. Pancreas 30: 218-222, 2005.

21. Singer EA, Gupta GN and Srinivasan R: Update on targeted therapies for clear cell renal cell carcinoma. Curr Opin Oncol 23: 283-289, 2011. 